\title{
Nonadiabatic ab initio molecular dynamics using linear-response time-dependent density functional theory
}

\author{
Basile F. E. Curchod ${ }^{1}$, Thomas J. Penfold ${ }^{1,2,3}$, Ursula Rothlisberger ${ }^{1}$, Ivano Tavernelli ${ }^{1 *}$ \\ 1 Laboratory of Computational Chemistry and Biochemistry \\ Ecole Polytechnique Fédérale de Lausanne, CH-1015 Lausanne, Switzerland \\ 2 Laboratoire de Spectroscopie Ultrarapide \\ Ecole Polytechnique Fédérale de Lausanne, CH-1015 Lausanne, Switzerland \\ 3 SwissFEL, Paul Scherrer Inst., $\mathrm{CH}-5232$ Villigen, Switzerland
}

Received 6 March 2013; accepted 24 October 2013

\begin{abstract}
:
We review our recent work on ab initio nonadiabatic molecular dynamics, based on linear-response timedependent density functional theory for the calculation of the nuclear forces, potential energy surfaces, and nonadiabatic couplings. Furthermore, we describe how nuclear quantum dynamics beyond the BornOppenheimer approximation can be performed using quantum trajectories. Finally, the coupling and control of an external electromagnetic field with mixed quantum/classical trajectory surface hopping is discussed.
\end{abstract}

PACS (2008): 31.10.+z, 31.15.ee, 71.15.Pd, 31.50.Gh

Keywords: $\quad$ nonadiabatic dynamics $\cdot$ time-dependent density functional theory $\cdot$ excited state $\cdot$ ab initio molecular dynamics

(c) Versita sp. z o.o.

\section{Introduction}

Within the Born-Oppenheimer approximation the coupling between nuclear and electronic motion is neglected. This approximation is behind most ground state electronic structure methods for molecules and led to the development of efficient mixed quantum/classical schemes for performing $a b$ initio molecular dynamics (AIMD) [1]. Within AIMD, the electrons are treated quantum mechanically and provide the potential for the classical dynamics of the nuclei. This gives rise to considerable computational

*E-mail: ivano.tavernelli@epfl.ch savings in comparison to the full quantum dynamics, especially when only one electronic state is considered, and as a result has been used to study a wide variety of medium to large molecular systems. However, as soon as more than one electronic state plays a role in the dynamics (for example for a photochemical reaction), the BornOppenheimer approximation will break down whenever the coupling between electronic states due to nuclear motion becomes important [2-4].

In this case, the resulting nonadiabatic nuclear dynamics can be accurately described using nuclear wavepacket propagation methodologies like, for example, multiconfiguration time-dependent Hartree (MCTDH) [5], but only for systems with a small number of active degrees of freedom. In addition, this method requires electronic properties like 
potential energy surfaces (PESs) to be computed before the propagation and their calculations can therefore become challenging for large systems.

On the other hand, the description of nonadiabatic events by means of trajectory-based approaches remains a challenge and only a few schemes are available. Among them, the trajectory surface hopping (TSH) method describes the nuclear wavepacket as a swarm of independent classical trajectories, which can jump from one electronic state to another whenever a region of strong nonadiabaticity is encountered along the dynamics $[6,7]$. TSH is able to correctly describe some nuclear quantum effects, such as the branching of the nuclear wavepackets among different electronic states. However, the independent trajectory approximation, central to the TSH method, directly implies that other nuclear quantum effects like (de)coherence or nuclear quantum tunneling remain inaccessible.

Quantum (or Bohmian) trajectories [8-10] constitute an interesting and alternative way to account for an accurate quantum propagation of the nuclei. Emerging from a transformation of the time-dependent (electronic-nuclear) Schrödinger equation (see Ref. [11] and Section 2.1.1 below), the quantum trajectory method is in principle exact and has been used to address challenging quantum dynamics problems in low dimensional model systems (see Ref. [12] for a complete description of quantum trajectory methods). Different quantum trajectory based approaches to nonadiabatic phenomena have been proposed (most of them based on a diabatic representation of the electronic states [13-16]) and we highlight in this article our method, in the adiabatic representation of the electronic states, called NonAdiabatic Bohmian DYnamics (NABDY) [1719].

Trajectory-based methods for nonadiabatic dynamics are indeed interesting as they are easily implemented within an on-the-fly scheme (where the electronic energies and nuclear forces are only required at the one point in coordinate space of the trajectory, at each time step). Coupling TSH with an efficient electronic structure method like linear-response time-dependent density functional theory (LR-TDDFT) [20-23] allows for the nonadiabatic dynamics of medium to large molecular systems. Alternatively, the use of a quantum trajectory based approach could con- stitute a way to perform on-the-fly nonadiabatic quantum dynamics. In this paper, we review our recent work on the development of efficient nonadiabatic methods coupled with LR-TDDFT. For an extended review on this topic, the interested reader is referred to the Ref. [24].

\section{Theory}

\subsection{Nonadiabatic dynamics}

\subsubsection{NonAdiabatic Bohmian DYnamics}

Starting from the time-dependent electron-nuclear Schrödinger equation for a molecular system (relativistic effects are not considered),

$$
\hat{H} \Psi(r, R, t)=i \hbar \frac{\partial}{\partial t} \Psi(r, R, t)
$$

we can use the Born-Huang expansion [25],

$$
\Psi(r, R, t)=\sum_{l}^{\infty} \Phi_{l}(r ; R) \Omega_{l}(R, t)
$$

to describe the total molecular wavefunction $\Psi(r, R, t)$. $r$ gives the positions of all the electrons of the system and $R$ those of the nuclei. $I$ denotes a specific electronic state, $\Omega_{l}(R, t)$ can be seen as a nuclear wavefunction, and $\Phi_{I}(r ; R)$ is a solution of the time-independent electronic Schrödinger equation,

$$
\hat{\mathcal{H}}_{e l} \Phi_{l}(\boldsymbol{r} ; \boldsymbol{R})=E_{l}^{e l} \Phi_{l}(\boldsymbol{r} ; \boldsymbol{R})
$$

After inserting the Born-Huang representation (Eq. (2)) into Eq. (1) and using the polar representation for a complex wavefunction

$$
\Omega_{\jmath}(R, t)=A_{\jmath}(R, t) \exp \left[\frac{i}{\hbar} S_{\jmath}(R, t)\right]
$$

we obtain equations of motion for the nuclear phases $S_{\jmath}(R, t) / \hbar$ and amplitudes $A_{\jmath}(R, t)$ ( $\gamma$ labels the nuclei),

$$
\begin{aligned}
& -\frac{\partial S_{J}(\boldsymbol{R}, t)}{\partial t}=\sum_{\gamma} \frac{1}{2 M_{\gamma}}\left(\nabla_{\gamma} S_{J}(\boldsymbol{R}, t)\right)^{2}+E_{J}^{e l}(\boldsymbol{R})-\sum_{\gamma} \frac{\hbar^{2}}{2 M_{\nu}} \frac{\nabla_{\gamma}^{2} A_{J}(\boldsymbol{R}, t)}{A_{J}(\boldsymbol{R}, t)}+\sum_{\gamma /} \frac{\hbar^{2}}{2 M_{\gamma}} D_{J l, \gamma}(\boldsymbol{R}) \frac{A_{l}(\boldsymbol{R}, t)}{A_{J}(\boldsymbol{R}, t)} \Re\left[e^{i \phi_{I J}(\boldsymbol{R}, t)}\right] \\
& -\sum_{\gamma, I \neq J} \frac{\hbar^{2}}{M_{\nu}} \boldsymbol{d}_{J l, \gamma}(\boldsymbol{R}) \frac{\nabla_{\gamma} A_{l}(\boldsymbol{R}, t)}{A_{J}(\boldsymbol{R}, t)} \Re\left[e^{i \phi_{I J}(\boldsymbol{R}, t)}\right]+\sum_{\gamma, I \neq J} \frac{\hbar}{M_{\gamma}} \boldsymbol{d}_{J l, \gamma}(\boldsymbol{R}) \frac{A_{l}(\boldsymbol{R}, t)}{A_{J}(\boldsymbol{R}, t)} \nabla_{\gamma} S_{l}(\boldsymbol{R}, t) \Im\left[e^{i \phi_{I J}(\boldsymbol{R}, t)}\right]
\end{aligned}
$$


and

$$
\begin{aligned}
& \frac{\partial A_{J}(\boldsymbol{R}, t)}{\partial t}=-\sum_{\gamma} \frac{1}{M_{\gamma}} \nabla_{\gamma} A_{\jmath}(\boldsymbol{R}, t) \nabla_{\gamma} S_{\jmath}(\boldsymbol{R}, t)-\sum_{\gamma} \frac{1}{2 M_{\gamma}} A_{\jmath}(\boldsymbol{R}, t) \nabla_{\gamma}^{2} S_{\jmath}(\boldsymbol{R}, t)+\sum_{\gamma /} \frac{\hbar}{2 M_{\gamma}} D_{J l, \gamma}(\boldsymbol{R}) A_{l}(\boldsymbol{R}, t) \Im\left[e^{i \phi_{I J}(\boldsymbol{R}, t)}\right] \\
& -\sum_{\gamma, l \neq J} \frac{\hbar}{M_{\gamma}} \boldsymbol{d}_{J l, \gamma}(\boldsymbol{R}) \nabla_{\gamma} A_{l}(\boldsymbol{R}, t) \Im\left[e^{i \phi_{I J}(\boldsymbol{R}, t)}\right]-\sum_{\gamma, l \neq J} \frac{1}{M_{\gamma}} \boldsymbol{d}_{J l, \gamma}(\boldsymbol{R}) A_{l}(\boldsymbol{R}, t) \nabla_{\gamma} S_{l}(\boldsymbol{R}, t) \Re\left[e^{i \phi_{I J}(\boldsymbol{R}, t)}\right],
\end{aligned}
$$

using the following definitions for $\phi_{I J}(R, t)$,

$$
\phi_{I J}(R, t)=\frac{1}{\hbar}\left[S_{I}(R, t)-S_{J}(R, t)\right]
$$

$H_{J I}(R)$,

$$
H_{J l}(R)=\int \Phi_{J}^{*}(r ; R) \hat{\mathcal{H}}_{e l} \Phi_{l}(r ; R) d r=\delta_{J l} E_{J}^{e l}(R),
$$

and the first and second order nonadiabatic couplings, respectively,

$$
\boldsymbol{d}_{J l, \nu}(\boldsymbol{R})=\int \Phi_{J}^{*}(r ; R) \nabla_{\nu} \Phi_{l}(r ; R) d r
$$

and

$$
D_{J I, \nu}(R)=-\int \Phi_{J}^{*}(r ; R) \nabla_{\nu}^{2} \Phi_{l}(r ; R) d r .
$$

Within the Hamilton-Jacobi formulation of mechanics, the relation between momentum and phase of the nuclear wavepacket,

$$
\nabla_{\beta} S_{J}(R, t)=P_{\beta}^{\prime},
$$

gives rise to a Newton-like equation for the nuclear motion (when $\nabla_{\beta}$ is applied on both sides of Eq. (5))

$$
\begin{aligned}
& M_{\beta} \frac{d^{2} R_{\beta}(t)}{\left(d t^{\jmath}\right)^{2}}= \\
& -\nabla_{\beta}\left[E_{e l}^{J}(R(t))+\mathcal{Q}_{J}(R(t), t)+\sum_{l} \mathcal{D}_{J l}(R(t), t)\right]
\end{aligned}
$$

for which we have employed the definition of the time derivative in a Lagrangian frame:

$$
d / d t^{\jmath}=\partial / \partial t+\sum_{\gamma} \nabla_{\gamma} S_{\jmath}(R, t) / M_{\nu} \cdot \nabla_{\gamma} .
$$

At this point, no approximation has been made in the derivation of this set of working equations (Eqs. (5), (6), (12)).

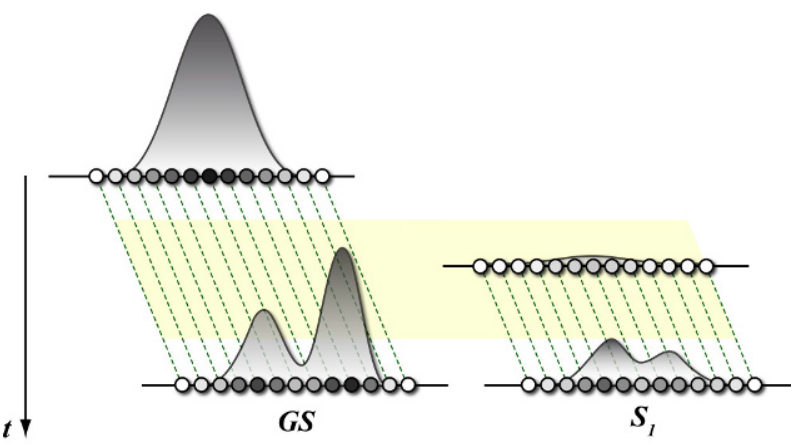

Figure 1. Schematic representation of the NonAdiabatic Bohmian DYnamics approach. Left (right): evolution of the nuclear wavepacket in the electronic ground state (electronic excited state). The vertical axis represents time and the light background indicates a region of strong nonadiabaticity between the two states.

Important for this work, Eq. (12) shows that, in addition to the "classical" potential $E_{e l}^{J}(R(t))$, the nuclei in trajectory $\boldsymbol{R}(t)$ feel the effect of a quantum potential $\mathcal{Q}_{\mathcal{J}}(\boldsymbol{R}(t), t)[12]$, which describes adiabatic nuclear quantum effects, and of nonadiabatic quantum potentials $\sum_{I} \mathcal{D}_{J I}(R(t), t)$ that are related to nonadiabatic effects between electronic states.

From a numerical point of view, the dynamics starts from an initial nuclear wavepacket at time $t=0$, which is "discretized" into a distribution of grid points called "fluid elements" (small volume elements of the configuration space, see Fig. 1). Each of these correlated fluid elements carries an amplitude, a phase, and a velocity. The integration of the NABDY equations of motion implies the simultaneous solution of the differential equations for the phases (Eq. (5)), the amplitudes (Eq. (6)), and the coupled Newton-like equations for the correlated fluid elements (Eq. (12)). During the initial adiabatic propagation of the quantum trajectories, nonadiabatic couplings are monitored. When their strength exceeds a given threshold, the nonadiabatic dynamics is initialized (Fig. 1). In this way, not only the trajectories on one electronic state are correlated, but also those among different electronic states. For numerical details, see Refs. [17-19]. 


\subsubsection{Trajectory surface hopping}

TSH $[6,7]$ is one of the most widely used (trajectorybased) methods for simulating nonadiabatic processes beyond the Born-Oppenheimer approximation. In practice, the initial nuclear wavepacket is portrayed by a swarm of independent classical trajectories, whose initial conditions (positions and momenta) are obtained, for example, from a thermal distribution in the ground electronic state (Fig. 2a). A set of time-dependent complex amplitudes, $\left\{C^{\alpha}(t)\right\}$ (one per electronic state I considered), is associated to each trajectory (labelled $\alpha$ ). These amplitudes are the time-dependent coefficients of the molecular wavefunction expansion ${ }^{1}$

$$
\Psi^{\alpha}(r, R, t)=\sum_{l}^{\infty} C_{l}^{\alpha}(t) \Phi_{I}(r ; R)
$$

in a complete basis of adiabatic electronic states $\Phi_{/}(\boldsymbol{r} ; \boldsymbol{R})$ (solutions of Eq. (3), with $R$ depending implicitly on time). Inserting Eq. (14) in the time-dependent Schrödinger equation for the electrons, multiplying on the left by $\Phi_{j}^{*}(\boldsymbol{r} ; \boldsymbol{R})$, and integrating over $\boldsymbol{r}$ leads to the following set of coupled equations for the amplitudes, associated with the different electronic states,

$$
i \hbar \dot{C}_{J}^{\alpha}(t)=\sum_{I} C_{l}^{\alpha}(t)\left(H_{J I}-i \hbar \dot{R}^{\alpha} \cdot \boldsymbol{d}_{J l}^{\alpha}\right)
$$

where $H_{\| l}$ and $\boldsymbol{d}_{J l}$ are described in the previous section. In the "Fewest-Switches" version of TSH [7], a classical trajectory $\alpha$ evolves adiabatically in a given electronic state according to Born-Oppenheimer dynamics until a hop between two adiabatic PESs $\left(H_{\|}=E_{l}^{e l}\right.$ and $H_{J J}=E_{J}^{e l}$ ) occurs (Fig. 2). The Fewest-Switches transition probability for such hop (from state $I$ to state $J$ in the time interval $[t, t+d t])$ is given by

$$
\begin{aligned}
& g_{l J}^{\alpha}(t, t+d t)= \\
& 2 \int_{t}^{t+d t} d \tau \frac{\Im\left[C_{J}^{\alpha}(\tau) C_{l}^{\alpha *} H_{J l}(\tau)\right]-\Re\left[C_{J}^{\alpha}(\tau) C_{l}^{\alpha *}(\tau) \Xi_{J l}^{\alpha}(\tau)\right]}{C_{l}^{\alpha}(\tau) C_{l}^{\alpha *}(\tau)}
\end{aligned}
$$

where $\Xi_{j l}^{\alpha}(\tau)=\dot{\boldsymbol{R}}^{\alpha} \cdot \boldsymbol{d}_{J /}^{\alpha}(\tau)$, and a hop occurs if and only if

$$
\sum_{K \leq J-1} g_{I K}^{\alpha}<\zeta<\sum_{K \leq J} g_{I K}^{\alpha}
$$

1 For an extended discussion on the TSH equations and their link with $N A B D Y$, the reader is referred to Ref [19, 24]. where $\zeta$ is a random number between 0 and 1 . The statistical distribution of a large number of such independent classical trajectories is assumed to mimic the probability density distribution of the corresponding nuclear wavepackets (Fig. 2b).

All matrix elements in Eqs. (15) and (16) are computed using a suitable $a b$ initio electronic structure method. In our implementation of TSH [26] we use density functional theory for the ground state and linear-response timedependent density functional theory for the excited state energies $E_{J}^{e l}(R)$, nuclear forces $F_{J}^{e l}$, nonadiabatic couplings $\dot{\boldsymbol{R}} \cdot \boldsymbol{d}_{J l}$, and nonadiabatic coupling vectors $\boldsymbol{d}_{J l}$ (see Refs. [26-30] for a detailed description of TSH implementation in the plane-wave software package CPMD [31]). In the next section we discuss the calculation of matrix elements for one-body operators within LR-TDDFT.

\subsection{Matrix elements of one-body operators within linear response time-dependent density functional theory}

In this section we briefly summarize how to use LRTDDFT to compute matrix elements of one-body operators such as the nonadiabatic coupling vectors (NACVs). The NACVs between two electronic states $J$ and $/$ for nucleus $\gamma\left(\boldsymbol{d}_{l,, \gamma}\right)$ are defined in terms of many-electron wavefunctions $\Phi$.

$$
\boldsymbol{d}_{J l, \gamma}=\left\langle\Phi_{J}\left|\nabla_{\gamma}\right| \Phi_{l}\right\rangle=\frac{\left\langle\Phi_{J}\left|\nabla_{\gamma} \hat{\mathcal{H}}_{e l}\right| \Phi_{l}\right\rangle}{E_{l}-E_{J}}
$$

where $\nabla_{\gamma}$ is the gradient with respect to the nucleus $\gamma$. It has been recently showed that matrix elements of a general one-body operator $\hat{\mathcal{O}}$ can be obtained within LRTDDFT using a set of auxiliary many-electron wavefunctions defined as (for a given excited electronic state I) [21]

$$
\left|\tilde{\Psi}_{1}\right\rangle=\sum_{i a} c_{i a}^{\prime} \hat{c}_{a}^{\dagger} \hat{c}_{i}\left|\tilde{\Psi}_{0}\right\rangle
$$

In Eq. (19), ia denotes a single excitation from the occupied Kohn-Sham (KS) orbital $i$ to the virtual orbital $a, \hat{c}_{a}^{+}$ $\left(\hat{c}_{i}\right)$ is the corresponding creation (annihilation) operator of KS orbitals, $\left|\tilde{\Psi}_{0}\right\rangle$ is the ground state KS Slater determinant, and $c_{i a}^{\prime}=\sqrt{\frac{\epsilon_{a}-\epsilon_{i}}{\Omega_{l}}} e_{i a}^{\prime}$ are coefficients for excited state $/$ that are entirely defined in terms of LR-TDDFT quantities: transition energy eigenvalues $\Omega$, with corresponding eigenvectors $e^{l}$, and KS orbital energy differences $\left(\epsilon_{a}-\epsilon_{i}\right)[28,30,32,33]$.

In LR-TDDFT, matrix elements of one-body operators involving the ground state $(0)$ and a given excited electronic 

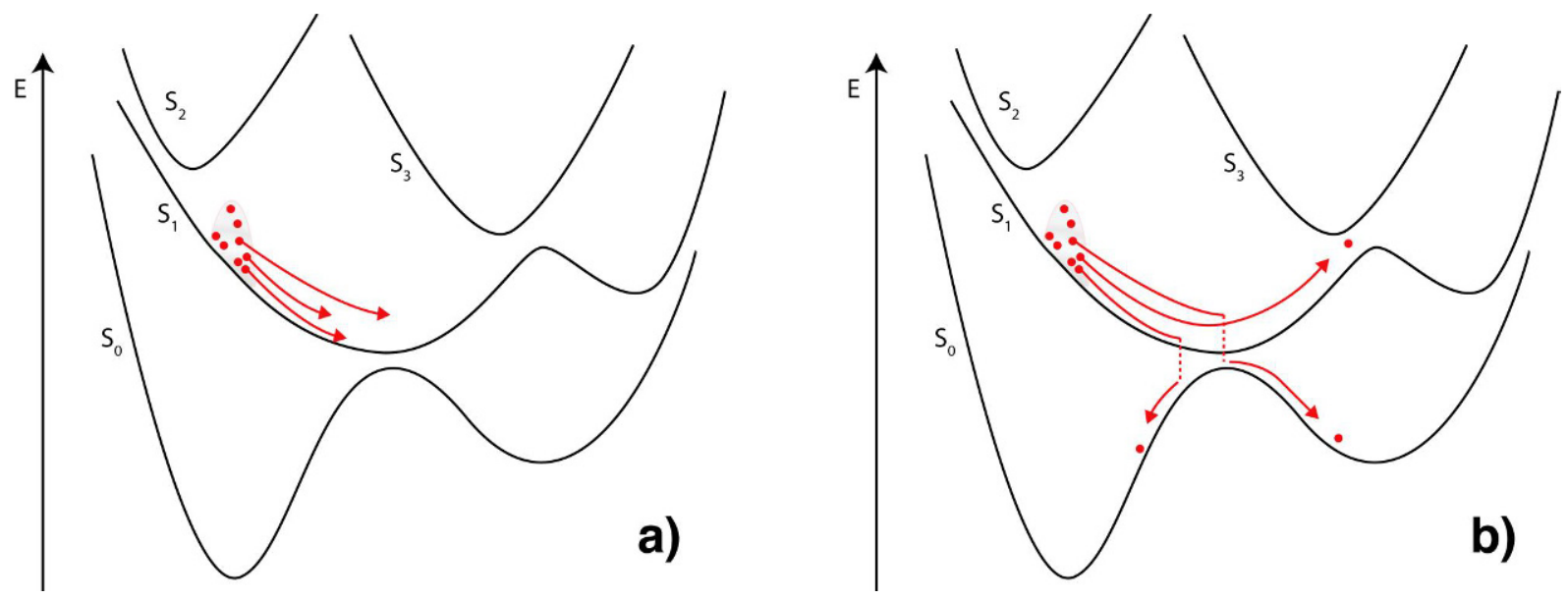

Figure 2. Schematic representation of TSH dynamics. a) The dynamics starts on the desired excited state from an initial distribution of trajectories (red dots). Each trajectory is time-evolved independently on an adiabatic potential energy surface. b) Whenever a region of strong nonadiabaticity is reached, the trajectory can hop following the probabilities given by Eq. (16).

state $(I)$ are obtained in the following way

$$
\begin{aligned}
\sigma_{0 l} & =\sum_{i a} c_{i a}^{\prime}\left\langle\tilde{\Psi}_{0}|\hat{\mathcal{O}}| \tilde{\Psi}_{i \rightarrow a}\right\rangle=\sum_{i a} c_{i a}^{\prime}\left\langle\phi_{i}|\hat{\mathcal{O}}| \psi_{a}\right\rangle \\
& =\sum_{i a} \sqrt{\frac{\epsilon_{a}-\epsilon_{i}}{\Omega_{l}}} e_{i a}^{l}\left\langle\phi_{i}|\hat{\mathcal{O}}| \psi_{a}\right\rangle,
\end{aligned}
$$

where $\tilde{\Psi}_{i \rightarrow a}$ represents an excited KS Slater determinant obtained from the one-electron excitation $i \rightarrow a$, and $\left\{\phi_{i}(r)\right\}_{i=1}^{N}\left(\left\{\psi_{a}(r)\right\}_{a=1}^{\infty}\right)$ are occupied (virtual) KS orbitals. For matrix elements of one-body operators involving two excited electronic states, $\sigma_{J l}$, the expression derived using the auxiliary many-electron wavefunctions corresponds exactly to the leading linear-response contribution obtained with the second-order coupled electronic oscillator approach of Ref. [34]. The expression becomes exact when the Tamm-Dancoff approximation (TDA) is used [35, 36].

\section{Applications}

In this section, we briefly present two applications based on the previously described methods.

\subsection{NonAdiabatic Bohmian DYnamics (NABDY)}

Here, we highlight our first application of NABDY to a simple nonadiabatic molecular process, the collision of an $\mathrm{H}$ atom with a $\mathrm{H}_{2}$ molecule. For this application, DFT and LR-TDDFT within the approximation of the LDA exchange-correlation functional have been used to compute PESs and nonadiabatic couplings on-the-fly, as

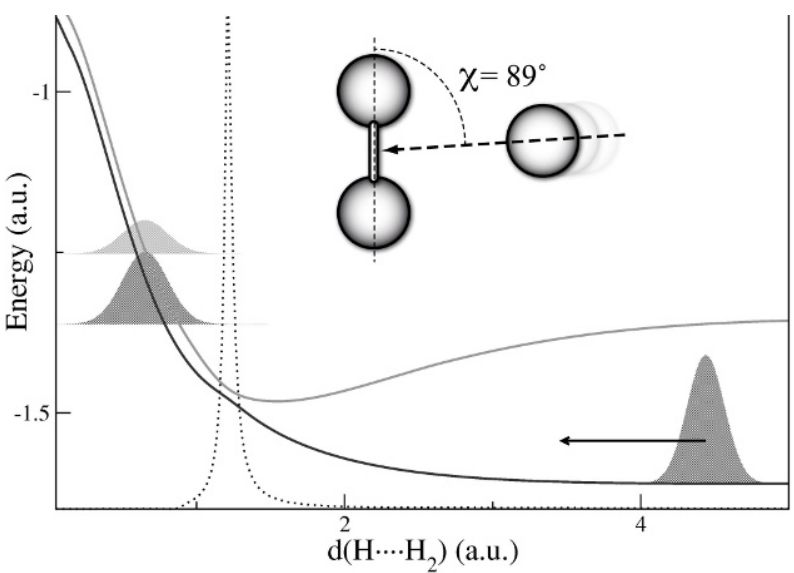

Figure 3. Collision of a $\mathrm{H}$ atom with a $\mathrm{H}_{2}$ molecule. PESs (dark grey: ground state, light grey: first excited state) and nonadiabatic couplings (black dotted line) are computed with DFT/LR-TDDFT. The initial nuclear wavepacket on the ground state is represented on the right. Inset depicts the system under study.

implemented in the plane-wave code CPMD (see Section 2.2). We discuss here a specific case (see Fig. 3), where the $\mathrm{H}$ atom is propagated in an almost perpendicular direction towards the $\mathrm{H}_{2}$ bond axis with the initial momentum $\left|P_{0}\right|=150$ a.u.. For such momentum, a population transfer of $31.4 \%$ to the first excited state is observed, due to a region of strong nonadiabaticity. Interested readers are invited to consult the references for more information and for the current challenges related to the implementation of NABDY $[18,19]$ and quantum trajectories methods in general [12]. 

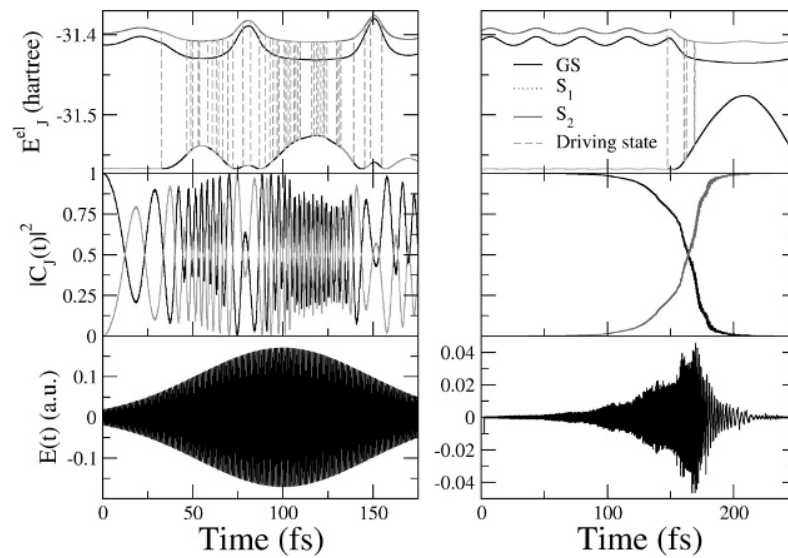

Figure 4. TSH dynamics of LiF coupled with two different timedependent external fields: left: П-pulse; right: local control pulse. Upper panel: Potential-energy curves along the dynamics obtained by LR-TDDFT/TSH calculations. Dashed gray line highlights the running state. Mid panel: Probabilities (square of the complex amplitudes) of each state. Lower panel: Applied external pulse.

\subsection{Local control theory within trajectory sur- face hopping}

In Section 2.1.2, we discussed the standard equations of trajectory surface hopping. A series of recent works have presented methods to couple the TSH equations with an external time-dependent electromagnetic field [29, 37-40]. Using these methodologies, it becomes possible to simulate light absorption (photoexcitation) of a molecular system and its subsequent nonadiabatic relaxation. A further development is to consider how a time-dependent electric field can be shaped such that it can control the population of a specific electronic excited state. In our recent work [41, 42], we have investigated the use of local control theory (LCT) [43] in the context of TSH/LR-TDDFT. Our implementation was tested on the diatomic molecule lithium fluoride (LiF). Using a strong and resonant $\Pi$ pulse, the dynamics based on TSH exhibits amplitude transfer from the ground state to the second excited singlet state $\left(S_{2}\right)$ of LiF. However, this transfer is not stable and large oscillations in amplitude are observed throughout the dynamics (Fig. 4, left). On the other hand, TSHbased LCT produces a shaped pulse that efficiently promotes the molecule into $S_{2}$ (Fig. 4, right) and shares some interesting similarities with a pulse calculated using LCT in combination with quantum nuclear dynamics [44] (see Ref. [41] for more details).

\section{Conclusions}

We have reviewed some of our recent work on nonadiabatic dynamics. TSH constitutes an efficient method for describing nonadiabatic processes and, when coupled with LR-TDDFT, can simulate photophysical and photochemical processes of medium to large size molecules. In addition, the central equations of TSH leading to surface hops are easily modified to include the coupling between the amplitudes and an external electromagnetic field. We have also discussed how matrix elements of one-body operators can be obtained rigorously from LR-TDDFT. Finally, we have presented a new method based on Bohmian dynamics, NABDY, that fully incorporates nuclear quantum effects in a trajectory-based nonadiabatic dynamics and is currently under active development.

\section{Acknowledgements}

The authors thank Giovanni Ciccotti, Sara Bonella, and Felipe Franco de Carvalho for their useful comments. COST action CM0702, Swiss NSF grant 200020-130082, and NCCR-MUST are acknowledged for fundings.

\section{References}

[1] D. Marx, J. Hutter, Ab Initio Molecular Dynamics: Basic Theory and Advanced Methods (Cambridge University Press, 2009)

[2] A. W. Jasper, S. Nangia, C. Zhu, D. G. Truhlar, Acc. Chem. Res. 39, 101 (2006)

[3] K. Takatsuka, T. Yonehara, Phys. Chem. Chem. Phys. 13, 4987 (2011)

[4] T. Yonehara, K. Hanasaki, K. Takatsuka, Chem. Rev. 112, 499 (2012)

[5] M. H. Beck, A. Jäckle, G. A. Worth, H. D. Meyer, Phys. Rep. 324, 1 (2000)

[6] J. C. Tully, R. K. Preston, J. Chem. Phys. 55, 562 (1971)

[7] J. C. Tully, J. Chem. Phys. 93, 1061 (1990)

[8] D. Bohm, Phys. Rev. 85, 166 (1952)

[9] D. Bohm, Phys. Rev. 85, 180 (1952)

[10] T. Takabayasi, Prog. Theor. Phys. 8, 143 (1952)

[11] P. R. Holland, The Quantum Theory of Motion - An Account of the de Broglie-Bohm Causal Interpretation of Quantum Mechanics (Cambridge University Press, 1993)

[12] R. E. Wyatt, Quantum dynamics with trajectories: Introduction to quantum hydrodynamics (Interdisciplinary applied mathematics, Springer, 2005) 
[13] R. E. Wyatt, C. L. Lopreore, G. Parlant, J. Chem. Phys. 114, 5113 (2001)

[14] C. L. Lopreore, R. E. Wyatt, J. Chem. Phys. 116, 1228 (2002)

[15] B. Poirier, G. Parlant, J. Phys. Chem. A 111, 10400 (2007)

[16] S. Garashchuk, V. A. Rassolov, G. C. Schatz, J. Chem. Phys. 123, 174108 (2005)

[17] B. F. E. Curchod, I. Tavernelli, U. Rothlisberger, Phys. Chem. Chem. Phys. 13, 3231 (2011)

[18] B. F. E. Curchod, U. Rothlisberger, I. Tavernelli, Chimia 66, 174 (2012)

[19] B. F. E. Curchod, I. Tavernelli, J. Chem. Phys. 138, 184112 (2013)

[20] E. Runge, E. K. U. Gross, Phys. Rev. Lett. 52, 997 (1984)

[21] M. E. Casida, in Recent Advances in Density Functional Methods, edited by D. P. Chong (Singapore, World Scientific, 1995), p. 155

[22] M. Petersilka, U. J. Gossmann, E. K. U. Gross, Phys. Rev. Lett. 76, 1212 (1996)

[23] H. Appel, E. K. U. Gross, K. Burke, Phys. Rev. Lett. 90, 043005 (2003)

[24] B. F. E. Curchod, U. Rothlisberger, I. Tavernelli, ChemPhysChem 14, 1314 (2013)

[25] M. Born and K. Huang, Dynamical Theory of Crystal Lattices (Clarendon, Oxford, 1954)

[26] E. Tapavicza, I. Tavernelli, U. Rothlisberger, Phys. Rev. Lett. 98, 023001 (2007)

[27] J. Hutter, J. Chem. Phys. 118, 3928 (2003)

[28] I. Tavernelli, E. Tapavicza, U. Rothlisberger, J. Mol. Struc. (Theochem) 914, 22 (2009)

[29] I. Tavernelli, B. F. E. Curchod, U. Rothlisberger, Phys. Rev. A 81, 052508 (2010)
[30] I. Tavernelli, B. F. E. Curchod, U. Rothlisberger, Chem. Phys. 391, 101 (2011)

[31] CPMD, Copyright IBM Corp 1990-2008, Copyright MPI für Festkörperforschung Stuttgart 1997-2001 (2011), http://www.cpmd.org

[32] I. Tavernelli, E. Tapavicza, U. Rothlisberger, J. Chem. Phys. 130, 124107 (2009)

[33] I. Tavernelli, B. F. E. Curchod, U. Rothlisberger, J. Chem. Phys. 131, 196101 (2009)

[34] S. Tretiak, S. Mukamel, Chem. Rev. 102, 3171 (2002)

[35] A. Fetter and J. Walecka, Quantum Theory of ManyParticle Systems (McGraw-Hill, New York, 1971)

[36] I. Tavernelli, B. F. E. Curchod, A. Laktionov, U. Rothlisberger, J. Chem. Phys. 133, 194104 (2010)

[37] M. Thachuk, M. Y. Ivanov, D. M. Wardlaw, J. Chem. Phys 105, 4094 (1996)

[38] G. A. Jones, A. Acocella, F. Zerbetto, J. Phys. Chem. A 112, 9650 (2008)

[39] R. Mitrić, J. Petersen, V. Bonačić-Koutecký, Phys. Rev. A 79, 053416 (2009)

[40] M. Richter, P. Marquetand, J. González-Vázquez, I. Sola, L. González, J. Chem. Theory Comput. 7, 1253 (2011)

[41] B. F. E. Curchod, T. J. Penfold, U. Rothlisberger, I. Tavernelli, Phys. Rev. A 84, 042507 (2011)

[42] B. F. E. Curchod, T. J. Penfold, U. Rothlisberger, I. Tavernelli, Chimia 67, 218 (2013)

[43] V. Engel, C. Meier, D. J. Tannor, Local Control Theory: Recent Applications to Energy and Particle Transfer Processes in Molecules (John Wiley \& Sons, Inc., 2009), pp. 29-101, ISBN 9780470431917

[44] T. J. Penfold, G. A. Worth, C. Meier, Phys. Chem. Chem. Phys. 12, 15616 (2010) 